\title{
PERFIL DE SENSIBILIDADE ANTIMICROBIANA DE CEPAS ESCHERICHIA COLI ISOLADAS DE INFECÇÕES URINÁRIAS COMUNITÁRIAS
}

\section{ANTIMICROBIAL SENSITIVITY PROFILE OF ESCHERICHIA COLI STRAINS ISOLATED FROM COMUNITARY URINARY INFECTIONS}

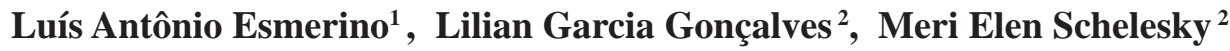 \\ 1 Autor para contato: Universidade Estadual de Ponta Grossa - UEPG, Campus em \\ Uvaranas, Departamento de Análises Clínicas e Toxicológicas, Ponta Grossa, PR, \\ Brasil; e-mail: esmerino@uepg.br; (42) 220-3113 \\ 2 Universidade Estadual de Ponta Grossa, Campus em Uvaranas, Departamento de \\ Farmácia da UEPG - (PIBIC), Ponta Grossa, PR, Brasil
}

Recebido para publicação em 28/04/2003

Aceito para publicação em 09/06/2003

\section{RESUMO}

O trato urinário é um dos locais mais comuns de infecção bacteriana e a Escherichia coli é o principal agente etiológico. Neste estudo, 58 cepas de Escherichia coli apresentaram o seguinte percentual de sensibilidade para os antimicrobianos do grupo A: ampicilina 37,9\%, cefazolina $56,1 \%$, cefalotina $77,2 \%$, gentamicina $96,6 \%$, nitrofurantoína $85,7 \%$, ofloxacina $94,6 \%$. Nossos resultados mostraram que $94,8 \%$ das cepas são sensíveis à pelo menos 3 antibióticos, entre os testados do grupo A, e nenhuma das cepas isoladas foi resistente a todos os antimicrobianos deste grupo. Para os antimicrobianos do grupo B: amoxicilina/ ácido clavulâmico 93,0\%, ampicilina/sulbactan 100\%, aztreonam 96,6\%, cefepima $96,6 \%$, cefoperazona $91,4 \%$, cefotaxima $94,8 \%$, cefoxitina $79,3 \%$, ceftazidima $96,6 \%$, ceftriaxona $96,6 \%$, cefuroxima $94,6 \%$, ciprofloxacina $93 \%$, cloranfenicol $72,4 \%$, tetraciclina $53,4 \%$ e tobramicina $100 \%$. Na triagem preliminar, 18 (31\%) das amostras foram caracterizadas como suspeitas de produzirem ESBL (betalactamase de espectro ampliado); a presença foi confirmada com o teste da adição de inibidores de betalactamases em discos de betalactâmicos, em duas cepas (3,5\%). Nossos estudos mostraram que a presença de cepas de Escherichia coli, isoladas de infecções urinárias comunitárias, produtoras de ESBL em nosso meio ainda não constitui um problema significativo. A sensibilidade para os principais grupos de antimicrobianos foi de 98,3\% para os aminoglicosídeos, 93,8\% para as fluoroquinolonas e 85,4\% para os betalactâmicos.

Palavras-chave: Escherichia coli, infecção urinária, antibiograma, aminoglicosídeo, fluoroquinolona, betalactâmico 


\begin{abstract}
The urinary tract is one of the most common places of bacterial infection, and Escherichia coli is the main etiologic agent. In this study, 58 strains of Escherichia coli presented the following percentage of sensitivity to antimicrobial group A': ampicillin 37.9\%; cefazolin 56.1\%; cephalotin 77.2\%; gentamicin $96.6 \%$; nitrofurantoin $85.7 \%$; ofloxacin $94.6 \%$. Results brought up that $94.8 \%$ of stumps are sensible to, at least, three antibiotics which had been tested in group A and neither of the isolated strains resistant to all the groups antimicrobials. The following results were found for antimicrobial group B': amoxicillin/clavulamic acid 93\%; ampicillin/sulbactam 100\%; aztreonam 96.6\%; cefepime 96.6\%; cefoperazone 91.4\%; cefotaxime $94.8 \%$; cefoxitin $79.3 \%$; ceftazidime $96.6 \%$ ceftriaxone $96.6 \%$ cefuroxime 94.6\%; ciprofloxacin 93\%; chloranfenicol 72.4\%; tetracycline 53.4\% and tobramicin $100 \%$. In a preliminary screening, 18 (31\%) samples were characterized as suspect of producing ESBL (extended-spectrum beta-lactamases), and the suspicion was confirmed by the additional test of beta-lactamase inhibitors in disks of betalactamic, in two stumps (3,5\%). Our studies showed that the presence of Escherichia coli strains, isolated from urinary infections, producing ESBL in our environment is not a significant problem yet.. The sensitivity to the main antimicrobial groups was $98.3 \%$ to aminoglycosides, $93.8 \%$ to fluoroquinolones and $85.4 \%$ to beta-lactam antibiotics.
\end{abstract}

Key words: Escherichia coli, urinary tract infection, susceptibility tests, fluoroquinolones, aminoglycosides, beta-lactam antibiotics

\section{Introdução}

O trato urinário é um dos locais mais comuns de infecção bacteriana. Infecções urinárias não complicadas, adquiridas na comunidade, estão entre as infecções mais comuns que acometem a maioria das mulheres. Nos Estados Unidos da América considera-se que essas infecções sejam responsáveis por mais de 8 milhões de consultas médicas anuais (WARREN et al., 1999; GUPTA et al., 2001a). Além disso, muitas mulheres apresentam infecção recorrente (RAZ et al., 2000).

As infecções são comumente causadas por bacilos Gram-negativos e estudos mostram que a Escherichia coli é a bactéria mais isolada nas uroculturas, com $75 \%$ a $90 \%$ dos casos. Staphylococcus saprophyticus com 5\% a 15\% dos casos (particularmente nas mulheres mais jovens) e Enterococcus e outras enterobactérias, tais como espécies de Klebsiella e Proteus mirabilis, são responsáveis por $5 \%$ a $10 \%$ dos casos. Embora bem menos estudado, o espectro de agentes que causam pielonefrites não complicadas, é parecido com aqueles que causam cistite aguda (GUPTA et al., 2001b).

Sendo originária da microbiota intestinal, a Escherichia coli coloniza a mucosa genital e pode penetrar no sistema urinário. Uma vez no sistema urinário, ela adere e coloniza a mucosa urogenital por meio de fímbrias e adesinas (WATANABE et al., 1991). Essa aderência faz com que a bactéria seja capaz de resistir à eliminação pelo fluxo urinário e, assim, ela coloniza a mucosa urogenital, causando uma bacteriúria significativa, geralmente $\geq 10^{5}$ bactérias $/ \mathrm{mL}$.

Embora a maioria das infecções seja de natureza aguda e de curta duração, elas contribuem para uma taxa significante de morbidade na população. As infecções graves podem resultar em perda da função renal e em seqüelas permanentes. Nas mulheres se faz distinção entre cistite, uretrite e vaginite, mas o trato urinário é contínuo e os sintomas geralmente se superpõem (MINS et al., 1995).

Segundo Goodman e Gilman (1996), podem 
ocorrer amplas variações na sensibilidade de diferentes cepas da mesma espécie bacteriana aos agentes antimicrobianos. Antes de escolher o fármaco, é essencial obter informações sobre o padrão de sensibilidade do microrganismo infectante. O sucesso da terapia com base em antimicrobianos depende, entre outros aspectos, do conhecimento da sensibilidade do agente etiológico in vitro.

Por outro lado, tem-se observado um aumento da resistência dos microrganismos aos antimicrobianos, como a produção de betalactamase de espectro ampliado (ESBL -Extended-Spectrum Beta-Lactmases). As cefalosporinas de amplo espectro, tais como a cefotaxima, ceftazidima e ceftriaxona, também conhecidas como cefalosporinas de terceira geração, inicialmente eficazes na terapêutica contra bactérias Gram-negativas, são inativadas pelas ESBL (JACOBY, MEDEIROS, 1991; BUSH et al., 1995).

As ESBL são enzimas, produzidas em muitos bacilos Gram-negativos e conferem resistência às penicilinas, às cefalosporinas e ao aztreonam. São freqüentemente detectadas em cepas de Klebsiella spp e Escherichia coli, no entanto, estão também sendo detectadas de forma crescente em muitas outras enterobactérias (TENOVER et al., 1999; TZELEPI et al., 2000).

Com a finalidade de orientar o médico na escolha do antimicrobiano é realizado o antibiograma da bactéria isolada no processo infeccioso. O método de disco difusão é o mais utilizado e tem sido o método de referência para se detectar a sensibilidade das bactérias aos antimicrobianos (BAUER et al., 1966). No entanto, com o aparecimento de novos mecanismos de resistência por parte dos microrganismos, este método tem mostrado falhas, principalmente para se detectar a resistência por produção de betalactamase (REIS et al., 1998).

Nesse aspecto estudos epidemiológicos sobre a resistência dos bacilos Gram- negativos, a determinação in vitro do perfil de sensibilidade aos antimicrobianos e a pesquisa de betalactamases, podem contribuir para uma terapia mais segura e eficaz. Esses estudos podem orientar o clínico na escolha do antimicrobiano enquanto se espera o resultado da cultura e do antibiograma.
Assim, no presente trabalho estudamos a sensibilidade e pesquisamos a produção de ESBL em cepas de Escherichia coli isoladas de infecções urinárias comunitárias, com a finalidade de traçar um perfil de sensibilidade para os antimicrobianos.

\section{Materiais e Métodos}

\section{Amostra}

Foram analisadas no presente estudo 58 cepas de Escherichia coli provenientes de infecções urinárias comunitárias. As culturas foram processadas inicialmente no Laboratório Universitário de Análises Clínicas, setor de microbiologia, órgão do Departamento de Análises Clínicas e Toxicológicas da UEPG, e no Laboratório Geral Alfredo Berger, do Instituto de Saúde de Ponta Grossa, durante o ano de 2001.

As cepas de Escherichia coli foram isoladas e identificadas através de métodos adotados nos laboratórios citados, constando de inoculação primária em meios de rotina e identificação utilizandose série bioquímica para enterobactérias.

\section{Antibiograma}

O antibiograma pelo teste de disco difusão em ágar se fundamenta na capacidade de difusão do antibiótico através de um meio sólido. Esse método é o mais empregado devido à sua fácil execução, à reduzida utilização de materiais e reagentes e ao baixo custo.

Um disco de papel de filtro é impregnado com determinada quantidade de antimicrobiano e aplicado à superfície do ágar pré-inoculado com a bactéria a ser testada. O crescimento do microrganismo é inibido (sensível) ou não (resistente) na presença do antibiótico contido no disco de difusão. A concentração do antibiótico no disco é padronizada com base na concentração inibitória mínima.

Nessa técnica foi utilizado o meio de cultura Ágar Mueller-Hinton (pH 7,2 - 7,4), distribuído em placas de $150 \mathrm{~mm}$ diâmetro (60 mL) ou $90 \mathrm{~mm}$ diâmetro (25 mL), ambas com espessura de $4 \mathrm{~mm}$.

O inóculo foi preparado pelo método da sus- 
pensão direta. Com auxílio de uma alça bacteriológica, 3 a 4 colônias da bactéria foram suspensas em solução salina estéril. Para acertar a turbidez da suspensão comparamos com padrão 0,5 da escala de McFarland, em espectrofotômetro a $580 \mathrm{~nm}$. A inoculação foi feita com um "swab” umedecido no inóculo e em seguida semeado de forma homogênea, sobre a superfície do meio. Os discos, com o antimicrobiano a ser testado, foram colocados com uma pinça, cerca de $3 \mathrm{~cm}$ de distância um do outro, sobre o ágar já inoculado (KONEMAN et al., 2001).

As placas foram incubadas a $35^{\circ} \mathrm{C}$ por $16-18$ horas e a leitura do diâmetro dos halos de inibição foi realizada com régua. Para interpretar os resultados foram utilizadas tabelas do NCCLS (2000), padronizadas em milímetros para disco difusão.

Para o controle de qualidade do antibiograma, com o objetivo de avaliar a metodologia e a qualidade dos discos, utilizou-se uma cepa de referência, Escherichia coli ATCC 25922.

\section{Antimicrobianos}

Os discos testados foram divididos em dois grupos, A e B, com base nos critérios adotados pelo NCCLS. No grupo A foram testados aqueles considerados de primeira escolha e os testados nos casos de infecção urinária (grupo U do NCCLS): ampicilina $10 \mu \mathrm{g}$, cefazolina $30 \mu \mathrm{g}$, cefalotina $30 \mu \mathrm{g}$, gentamicina $10 \mu \mathrm{g}$, nitrofurantoína $300 \mu \mathrm{g}$, ofloxacina $5 \mu \mathrm{g}$. No grupo B foram testados aqueles que sempre devem ser testados, mas relatados somente em casos de resistência no grupo A, ou quando o paciente é alérgico aos fármacos de primeira escolha: amoxacilina/ácido clavulânico 20/ $10 \mu \mathrm{g}$, ampicilina/sulbactan 10/10 $\mu \mathrm{g}$, aztreonam $30 \mu \mathrm{g}$, cefepima $30 \mu \mathrm{g}$, cefoperazona $75 \mathrm{mg}$, cefotaxima $30 \mu \mathrm{g}$, cefoxitina $30 \mu \mathrm{g}$, ceftazidima 30 $\mu \mathrm{g}$, ceftriaxona $30 \mu \mathrm{g}$, cefuroxima $30 \mu \mathrm{g}$, ciprofloxacina $5 \mu \mathrm{g}$, cloramfenicol $30 \mu \mathrm{g}$, tetraciclina $30 \mu \mathrm{g}$ e tobramicina $10 \mu \mathrm{g}$.

Pesquisa de betalactamase de espectro ampliado (ESBL)

Teste de triagem

No teste de disco-difusão (antibiograma) amostras de Escherichia coli que apresentaram halos de inibição $\leq 22$ mm para cefpodoxima, $\leq 27$ mm para aztreonam, $\leq 27 \mathrm{~mm}$ para cefotaxima, $\leq 25 \mathrm{~mm}$ para ceftriaxona ou $\leq 22 \mathrm{~mm}$ para ceftazidima, foram consideradas suspeitas de produzirem ESBL (NCCLS, 2000). Recomenda-se que seja realizado o teste confirmatório, como o teste de adição de inibidores de betalactamase em discos de betalactâmicos.

\section{Teste confirmatório}

O teste confirmatório da produção dessa enzima foi realizado com a mesma técnica do antibiograma, só que utilizando discos de cefalosporinas com e sem ácido clavulânico. Foram utilizados discos de $30 \mu \mathrm{g}$ de ceftazidima e cefotaxima com e sem $10 \mu \mathrm{g}$ de ácido clavulânico. Os resultados da produção de ESBL são considerados positivos se após a leitura das placas, incubadas a $35^{\circ} \mathrm{C}$ por 18 horas, a diferença de tamanho dos halos obtidos no disco combinado e no disco da cefalosporina correspondente for de $\geq 5 \mathrm{~mm}$. Os resultados são considerados negativos se essa diferença for $<5 \mathrm{~mm}$ (NCCLS, 2000).

\section{Resultados}

Com relação à pesquisa de betalactamase, das 58 cepas de Escherichia coli analisadas, foram caracterizadas na triagem preliminar 18 (31\%) amostras suspeitas de serem produtoras de ESBL. Destas, 7 mostraram halos menores característicos de cepas produtoras de ESBL para aztreonam, 5 para cefotaxima, 2 para ceftriaxona, 1 para aztreonam e ceftazidima, 2 para cefotaxima e ceftazidima e 1 para aztreonam, cefotaxima e ceftazidima.

A Tabela 1 mostra o diâmetro médio dos halos de inibição obtidos no teste de triagem para a pesquisa de betalactamase de espectro ampliado (ESBL). As cepas consideradas negativas na triagem apresentaram halos de inibição maiores que $\mathrm{BR}$, o ponto de corte estabelecido pelo NCCLS.

A presença de ESBL foi confirmada com o disco de cefotaxima, combinado com ácido clavulânico, em duas cepas (3,5\%). 
Tabela 1 - Diâmetro médio em milímetros dos halos obtidos no teste de triagem para pesquisa de ESBL. Cepas com halos $\leq$ BR são consideradas suspeitas de produzirem ESBL. S (Sensível)

\begin{tabular}{c|ccccc}
\hline ANTIMICROBIANOS & S* $^{*}$ & BR $^{*}$ & ESBL - & ESBL + & TOTAL \\
\hline Aztreonam $30 \mu$ & $\geq 22$ & $\leq 27$ & $31,7(49-84,5 \%)$ & $25,9(9-15,5 \%)$ & $(58-100 \%)$ \\
Cefotaxima $30 \mu$ & $\geq 23$ & $\leq 27$ & $30,9(50-86,2 \%)$ & $25,4(8-13,8 \%)$ & $(58-100 \%)$ \\
Cftriaxona $30 \mu$ & $\geq 21$ & $\leq 25$ & $30,4(56-96,5 \%)$ & $23,0(2-3,5 \%)$ & $(58-100 \%)$ \\
Cftazidima $30 \mu$ & $\geq 18$ & $\leq 22$ & $27,2(55-94,8 \%)$ & $21,7(3-5,2 \%)$ & $(58-100 \%)$ \\
\hline
\end{tabular}

* Diâmetros em milímetros (Critérios do NCCLS, 2000)

O perfil de sensibilidade das linhagens de Escherichia coli isoladas de infecções urinárias comunitárias para os antimicrobianos do grupo A, já considerando a pesquisa de ESBL, pode ser observado na Tabela 2.

Com relação aos antibacterianos do grupo $\mathrm{A}$, observou-se que 14 cepas $(24,1 \%)$ se mostraram sensíveis a todos os seis antibacterianos testados. As demais cepas apresentaram a seguinte resistência: 18 cepas (31\%) para um antibacteriano, 15 $(25,9 \%)$ para dois, $8(13,8 \%)$ para três, $1(1,7 \%)$ para quatro e 2 (3,5\%) para cinco. Nenhuma das cepas apresentou resistência a todos os antibacterianos do grupo $\mathrm{A}$.

Tabela 2 - Perfil de sensibilidade e resistência aos antimicrobianos de cepas urinárias de Escherichia coli.

\begin{tabular}{l|c|c}
\hline \multirow{2}{*}{ ANTIMICROBIANOS } & \multicolumn{2}{|c}{ Escherichia coli } \\
\cline { 2 - 3 } GRUPO A & SENSIBILIDADE & RESISTENNCIA \\
AMPICILINA & & \\
CEFAZOLINA & $37,9 \%$ & $62,1 \%$ \\
CEFALOTINA & $56,1 \%$ & $43,9 \%$ \\
GENTAMICINA & $77,2 \%$ & $22,8 \%$ \\
NITROFURANTOÍNA & $96,6 \%$ & $3,4 \%$ \\
OFLAXACIN & $85,7 \%$ & $14,3 \%$ \\
\hline GRUPO B & $94,6 \%$ & $5,4 \%$ \\
AMICACINA/AC.CAVULÂMICO & $93,0 \%$ & $7 \%$ \\
AMPICILINA/SULBACTAM & $100 \%$ & $0 \%$ \\
AZTREONAN & $96,6 \%$ & $3,4 \%$ \\
CEFEPIMA & $96,6 \%$ & $3,4 \%$ \\
CEFOPERAZONA & $91,4 \%$ & $8,6 \%$ \\
CEFOTAXIMA & $94,8 \%$ & $5,2 \%$ \\
CEFOXITINA & $79,3 \%$ & $20,7 \% *$ \\
CEFTRIAXONA & $96,6 \%$ & $3,4 \%$ \\
CEFUROXIMA & $96,6 \%$ & $3,4 \%$ \\
CIPROFLOXACIN & $93,0 \%$ & $7 \%$ \\
CLORANFENICOL & $72,4 \%$ & $27,6 \%$ \\
TETRACICLINA & $53,4 \%$ & $46,6 \%$ \\
TOBRAMICINA & $100 \%$ & $0 \%$ \\
\hline
\end{tabular}

* 12,1\% das cepas apresentaram sensibilidade intermediária para Cefoxitina 
O perfil de sensibilidade para os antimicrobianos do grupo $\mathrm{B}$, já considerando a pesquisa de ESBL, pode ser observado na Tabela 2.

A sensibilidade geral para os principais grupos de antimicrobianos foi de $98,3 \%$ para os aminoglicosídeos, 93,8\% para as fluoroquinolonas e 85,4\% para os betalactâmicos (Figura 1). Com relação aos betalactâmicos, a sensibilidade geral foi maior entre os testados do grupo B $(93,9 \%)$ do que entre os do grupo A (57,1\%).

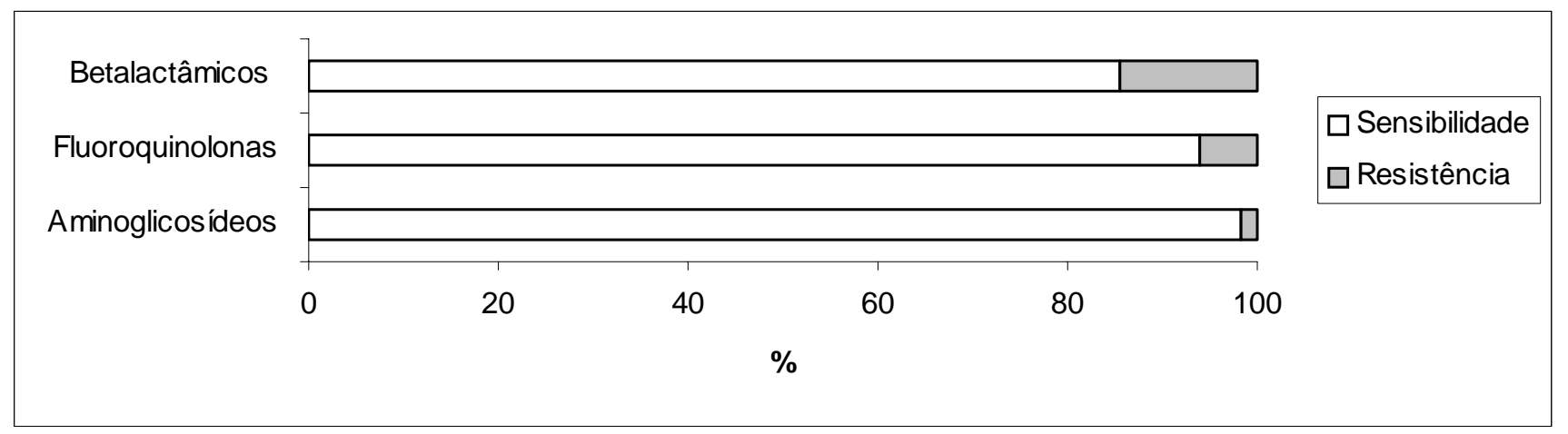

Figura 1 - Sensibilidade e resistência antimicrobiana de Escherichia coli isoladas de infecções urinárias comunitárias para os principais grupos de antimicrobianos testados.

\section{Discussão e Conclusão}

Embora o espectro dos agentes que causam infecções urinárias comunitárias tenha permanecido relativamente constante, sendo a Escherichia coli o seu principal agente, estudos demonstram um aumento na resistência dos uropatógenos isolados em culturas (HOOTON, STAMM, 1997).

Até mesmo em infecções urinárias agudas, não complicadas, em mulheres, tem sido observado um aumento na resistência da Escherichia coli para a ampicilina de $30 \%$ a $40 \%$, à cefalotina de $20 \%$ a $30 \%$ e ao trimetoprim-sulfametaxazol de 15\% a 20\% (STAMM, NORRBY, 2001).

As infecções do trato urinário estão entre as infecções bacterianas mais comuns nas mulheres. Terapia para essas infecções é usualmente iniciada antes que o resultado do antibiograma seja conhecido. Além disso, nas mulheres com cistite aguda não complicada, pré-terapia empírica, sem uma cultura de urina, é freqüentemente utilizada. Esse tratamento é baseado no conhecimento do espectro dos agentes etiológicos que causam as infecções do trato urinário, e seus modelos de resistência para os antimicrobianos. Entretanto, a resistência dos uropatógenos, tanto nas cistites como nas pielo- nefrites, está se modificando (GUPTA et al., 2001b).

Os testes para a detecção da sensibilidade são realizados em bactérias isoladas de amostras clínicas representativas de um processo infeccioso. Dessa forma, a avaliação da resistência aos antimicrobianos é uma das principais funções do laboratório de análises clínicas, especialmente do setor de microbiologia clínica (OPLUSTIL et al., 2000).

A crescente resistência aos antimicrobianos, observada para muitos gêneros de bactérias, faz com que os laboratórios de análises clínicas reavaliem suas metodologias de testes de sensibilidade e investiguem a presença dos novos mecanismos de resistência, como a produção de betalactamase.

A lista dos antimicrobianos que devem ser testados e reportados pelo laboratório, depende das características dos pacientes atendidos pelo mesmo e do perfil de sensibilidade das cepas normalmente isoladas. Além disso, novos antimicrobianos são lançados no mercado todos os anos fazendo com que os laboratórios necessitem rever suas listas freqüentemente.

No nosso estudo, os antimicrobianos do grupo A que apresentaram melhor sensibilidade foram a gentamicina (96,6\%), a ofloxacina $(94,6 \%)$ e a nitrofurantoína $(85,7 \%)$. A sensibilidade para o 
sulfazotrim, um antimicrobiano do grupo A, não foi determinada neste estudo, mas em um outro semelhante com 43 cepas de Escherichia coli, também isoladas de infecções comunitárias, a sensibilidade foi de 55,8\% (BAIL et al., 2002).

Nossos resultados mostraram que $94,8 \%$ das Escherichia coli, isoladas das infecções urinárias comunitárias, são sensíveis a pelo menos 3 antibióticos, entre os testados do grupo A, e nenhuma das cepas isoladas foi resistente a todos os antimicrobianos desse grupo. Desta forma, podemos verificar que a Escherichia coli, o principal agente etiológico das infecções urinárias comunitárias, é ainda bem sensível aos antimicrobianos considerados de primeira escolha.

Baseados nestes dados, os antimicrobianos do grupo B poderiam não ser relatados em muitos antibiogramas, mas como na maioria dos casos, não temos informações clínicas suficientes dos pacientes, relatamos a sensibilidade também para esses antimicrobianos, com a finalidade de aumentar a opção terapêutica. No entanto, deve-se estimular e incentivar o uso dos antimicrobianos de primeira escolha e reservar os de segunda para os casos mais graves.

Com relação aos antimicrobianos do grupo $\mathrm{B}$, de segunda escolha, todas as cepas foram sensíveis à ampicilina/sulbactam e à tobramicina. Geralmente as cepas produtoras de ESBL são resistentes à tobramicina, fato que não foi observado nas nossas duas cepas produtoras.

O grupo de antimicrobianos com melhor índice de sensibilidade foi o dos aminoglicosídeos 98,3\% (tobramicina 100\% e gentamicina 96,6\%). Esses antimicrobianos mostram-se eficazes contra muitos microrganismos aeróbicos Gram-negativos e alguns Gram-positivos e são mais utilizados contra microrganismos Gram-negativos entéricos (RANG et al., 2001).

As cepas de E. coli, no nosso estudo, se mostraram bastantes sensíveis in vitro aos antimicrobianos do grupo das fluoroquinolonas $(93,8$ $\%)$. Para ofloxacina e ciprofloxacina a sensibilidade foi de 94,6 \% e 93 \% (resistência de 5,4 \% e 7 $\%)$, respectivamente. A sensibilidade observada foi maior do que a relatada em outros estudos (GA-
LES et al, 2000).

A sensibilidade aos antimicrobianos do grupo dos betalactâmicos foi de 85,4\% (Figura 1) e a Escherichia coli foi mais sensível àqueles antimicrobianos incluídos no grupo B do que àqueles do grupo A (Tabela 2). Duarte et al. (2002) estudaram a infecção urinária em gestantes e observaram baixos percentuais de sensibilidade para a ampicilina (60,6\%) e cefalotina (63,6\%) e altos percentuais de sensibilidade para a cefuroxima (95,5\%). Os autores destacam a necessidade de avaliação periódica do padrão de sensibilidade dos agentes etiológicos prevalecentes aos antimicrobianos e recomendam o uso da cefuroxima como o antimicrobiano de escolha para o tratamento das infecções do trato urinário em gestantes.

No caso de uma cepa de Escherichia coli ser ESBL positiva, o resultado do antibiograma deve ser reportado como: “cepa produtora de ESBL”. O antibiograma deve ser relatado como resistente a todas as penicilinas, todas as cefalosporinas e todas as monobactam, independentemente do resultado obtido no estudo in vitro (OPLUSTIL et al., 2000).

A dificuldade na detecção de cepas produtoras de ESBL é decorrente de vários fatores, como a grande variedade de tipos de ESBL, que podem variar de cepa para cepa e alterações na potência e afinidade das betalactamases aos diferentes antimicrobianos betalactâmicos. A detecção laboratorial apresenta grande importância, uma vez que há vários relatos de falência terapêutica devido ao uso de cefalosporinas de terceira geração e penicilinas de amplo espectro no tratamento de infecções causadas por cepas produtoras de ESBL (REIS et al, 2001). Com a finalidade de aumentar a detecção, os laboratórios poderiam utilizar diferentes métodos, como: técnicas de disco aproximação, E-test e a adição de inibidores de betalactamase em discos de cefalosporinas. Essa última técnica é adotada pelo NCCLS.

A partir dos nossos resultados podemos observar que dentro desta amostragem a incidência de linhagens de Escherichia coli, isoladas em infecção urinárias comunitárias, produtoras de ESBL em nosso meio é ainda pequena $(3,5 \%)$ e não cons- 
titui um problema significativo. Outros estudos mostraram resultados semelhantes. No entanto, é necessário que os laboratórios de análises clínicas estejam atentos e preparados para que não ocorra uma sub-notificação do problema (HONORIO et al., 2001). Dessa forma, o monitoramento da resistência e o estudo do perfil de sensibilidade das bactérias patogênicas em uma determinada região, pode auxiliar os clínicos, farmacêuticos e demais profissionais da saúde no acompanhamento terapêutico e na orientação dos pacientes.

\section{REFERÊNCIAS}

1 BAIL, L.; ITO, C.A.S.; ESMERINO, L.A. Perfil de sensibilidade antimicrobiana para enterobactérias isoladas em infecção urinária. In: Encontro de Pesquisa da UEPG, 2., 2002. Ponta Grossa.

2 BAUER, A.W.; KIRBY, W.M; SHERRIS, J.C.; TURCK, M. Antibiotic susceptibility testing by a standardized single disk method. Am. J. Clin. Pathol. v. 45, p. 493-6, 1966.

3 BUSH, K.; JACOBY, G.A.; MEDEIROS, A .A. A functional classification scheme for b-lactamase and its correlation with molecular struture. Antimicrob. Agents Chemother. v. 39, p. 1211-1233, 1995.

4 DUARTE, G.; MARCOLIN, A. C.; GONÇALVES, C. V. Infecção urinária na gravidez: análise dos métodos para diagnóstico e do tratamento. Rev. Bras. Ginecol. Obstet. v. 24, n. 7, p. 471-477, 2002.

5 GALES, A. C.; JONES, R. N.; GORDON, K. A.; SADER, H. S.; WILKE, W. W.; BEACH, M. L.; PFALLER, M. A.; DOERN, G. V. and the SENTRY Study Group Latin America. Activity and spectrum of 22 antimicrobial agents tested against urinary tract infection pathogens in hospitalized patients in Latin America: report from the second year of the SENTRY Antimicrobial Surveillance Program (1998) J. Antimicrob. Chemother. v. 45, p. 295-303, 2000.

6 GOODMAN, L. S.; GILMAN, A. GODMAN \& GILMAN'S the pharmacological basis of therapeutics. 9. ed. New York: MacGraw-Hill, 1996.

7 GUPTA, K.; HOOTON, T. M.; ROBERTS, P. L.; STAMM, W. E. Patient-initiated treatment of uncomplicated recurrent urinary tract infections in young women. Ann. Intern. Med. v. 135 , n. 1, p. 9-16, 2001a.
8 GUPTA, K.; HOOTON, T. M.; STAMM, W. E. Increasing antimicrobial resistance and the management of uncomplicated community-acquired urinary tract infections. Ann. Intern. Med. v. 135, n. 1, p. 41-50, 2001b.

9 HONÓRIO, L. C.; SANTOS, I. B.; ASSIS, A. M. L.; SANTOS FILHO, L. Análise do perfil de resistência de enterobactérias produtoras de b-lactamases de espectro ampliado (ESBL) isoladas em João Pessoa, PB. Rev. Bras. de Análises Clínicas. v. 33. n. 4, p. 179-182, 2001.

10 HOOTON, T. M.; STAMM, W. E. Diagnosis and treatment of uncomplicated urinary tract infection. Infect. Dis. Clin. North. Am. v. 11, n. 3, p. 551-558, 1997.

11 JACOBY, G. A.; MEDEIROS, A. A. More extendedspectrum b-lactamases. Antimicrob. Agents Chemother. v. 35, p. 1697-1714, 1991.

12 KONEMAN, E. W.; ALLEN, S. D.; JANDA, W. A.; SCHRECKENBERGER, P. C.; WINN, W. C. Diagnóstico Microbiológico - Texto e Atlas Colorido. 5. ed. Rio de Janeiro: Medsi, 2001.

13 MINS, A. C. ; PLAYFAIR, J. HL. ; ROIT, I. M.; WAKELIN, D.; WILLIAMS, R. Microbiologia médica. 1. ed. São Paulo: Manole, 1995.

14 NCCLS (NATIONAL COMMITTEE FOR CLINICAL LABORATORY STANDARD) Performance Standards for Antimicrobial Susceptibility Testting: Twelfth Informational Supplement. Copyright The National Committee for Clinical Laboratory Standards, Wayne, PA, 2000.

15 RANG, H. P.; DALE, M. M.; RITTER, J. M. Farmacologia. 4. ed. Rio de Janeiro: Guanabara-Koogan, 2001.

16 RAZ, R.; GENNESIN, Y.; WASSER, J.; STOLER, Z.; ROSENFELD, S.; ROTTENSTERICH, E.; STAMM, W. E. Recurrent urinary tract infections in postmenopausal women. Clin. Infect. Dis. v. 30, p. 152-156, 2000.

17 REIS, A. O.; GALES, A. C.; MIRANDA, E. A.; SADER, H. S. Avaliação da acurácia do teste de adição de clavulanato em disco para a detecção de amostras de Klebsiella Pneumoniae produtoras de b-lactamases de espectro ampliado (ESBL). J. Bras. Patol. v. 34, n. 2 p. 85-92, 1998.

18 REIS, A. O.; GALES, A. C.; SADER, H. S. Detecção de b-lactamases de espectro ampliado (ESBL) pelo laboratório clínico: uma revisão crítica. J. Bras. Patol. v. 37 n. 1 p. 4348, 2001.

19 STAMM, W. E.; NORRBY, S. R. Urinary tract infections: Disease panorama and challenges. J. Infect. Dis. v. 183, s1s4, 2001. 
20 TENOVER, F. C.; MOHAMMED, M. J.; GORTON, T. S.; DEMBEK, Z. F. Detection and reporting of organisms producing extended-spectrum b-lactamases: survey of laboratories in Connecticut. J. Clin. Microbiol. v. 37, n. 12, p. 4065-4070, 1999.

21 TZELEPI, E.; GIAKKOUPIG, P.; SOFIANOU, D.

Detection of extended-spectrum beta-lactamases in clinical isolates of Enterobacter cloacae and Enterobacter aerogenes J. Clin. Microbiol. v. 38, n. 2, p. 542-546, 2000.

22 WARREN, J.W.; ABRUTYN, E.; HEBEL, J.R.;
JOHNSON, E.; SCHAEFFER, A. J.; STAMM, W. E. Guidelines for antimicrobial treatment of uncomplicated acute bacterial cystitis and acute pyelonephritis in women. Infectious Diseases Society of America (IDSA). Clin. Infect. Dis. v. 29, n. 7, p. 45-58, 1999.

23 WATANABE, D. S. A.; MICHELIN, L. A.; MONTELLI, A. C. Infecção urinária por Escherichia coli - correlação de características clínicas, patogênicas e sorogrupos. Rev. Bras. Patol. Clin. v. 27, n. 4, p. 111-117, 1991 